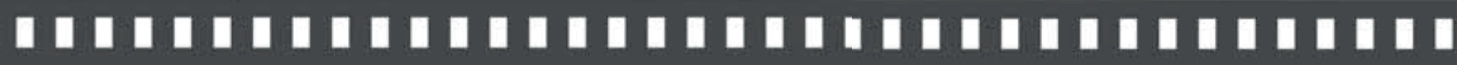

\author{
Madridimagen: tras las huellas del \\ último festival de cine de fotografía
}

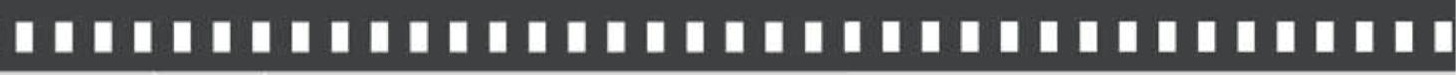

Montserrat Jurado Martín e Laura Cortés Selva

Artigo recebido em: 20/04/2018

Artigo aprovado em: 03/09/2018

D0I 10.5433/1984-7939.2018v14n25p220 


\section{Madridimagen: tras las huellas del último festival de cine de fotografía}

Madridimagen: following the footsteps of the last photographic film festival

Montserrat Jurado Martín*

Laura Cortés Selva**

Resumo: Este artículo rescata la historia de Madridimagen (1996-2012), el único festival de cine especializado en fotografía en España. Se demuestra que cumplía con su función de promotor de los directores de fotografía, que aumentaron su producción y presencia tras el reconocimiento recibido en el certamen. Su desaparición no queda justificada desde el ámbito cultural y tras los análisis documental y biográfico se demuestra que su final estuvo relacionado con cuestiones extra culturales relacionadas con la identidad ideológica de sus patrocinadores.

Palavras-chave: Festival de cine. Cultura. Dirección de fotografia. Industria cultural.

Abstract: This article rescues the history of Madridimagen (1996-2012), the only film festival specializing in photography in Spain. It is shown that it fulfilled its role as promoter of the directors of photography, who increased their production and presence after the recognition received in the contest. Its disappearance is not justified from the cultural sphere and after the documentary and biographical analysis it is demonstrated that its end was related to extra cultural issues related to the ideological identity of its sponsors.

Keywords: Film festival. Culture. Direction of photography. Cultural industry.

* Doctora. Profesora del Departamento de Ciencias Sociales y Humanas de la Universidad Miguel Hernández de Elche.

** Doctora. Profesora del Departamento de Comunicación Audiovisual de la Universidad Católica de Murcia. 


\section{Introducción $^{1}$}

Los festivales de cine son agentes activos en la consolidación de los profesionales en ciernes del sector cinematográfico, ya que son espacios donde las nuevas promesas se inician en la exhibición de sus trabajos. Como escaparate comercial de la industria cinematográfica, es el lugar idóneo para que el autor pueda probar su aptitud previo a la inversión en un largometraje en la gran pantalla, para que productores del sector y nuevas promesas inicien contactos, dar a conocer nuevas técnicas, etc.

La literatura relacionada con los festivales de cine es principalmente de origen anglosajón y todavía tiene camino por andar. Los estudios de referencia están representados por las publicaciones anuales de Iordanova en colaboración con otros investigadores (IORDANOVA; RHYNE, 2009; IORDANOVA; CHEUNG， 2010， 2011; IORDANOVA; LESHU, 2012; IORDANOVA; PEER, 2014). Por una parte, la autora reflexiona sobre la relación entre la política y la economía y, por otra, el fenómeno cultural y social y las trasformaciones que se establecen en el contexto de los festivales de cine. Destacan también los trabajos de De Valck (2007), que, aunque se centren en el estudio de los principales certámenes europeos, sus conclusiones pueden

1 Atendiendo a la igualdad de género, en este artículo se ha recurrido a la terminología neutra de la lengua española para la descripción de sustantivos o determinantes que acompañan a sustantivos que impliquen al mismo tiempo a hombres y mujeres. De este modo palabras como profesor, el docente o los investigadores, hacen referencia a profesor/a, la/el docente o las/los investigadora/ os. 
ser aplicadas al resto, ya que analizan los criterios que fomentan su consolidación. De Valck, Kredell y Loist (2016) estudian los festivales de cine a nivel mundial fijando su atención en la historia y su descripción. Turan (2002) ofrece una perspectiva más genérica frente a la propuesta de definición teórica de Stringer (2003).

En el caso español destacan los estudios sobre festivales de cine de Aida Vallejo $(2012,2014)$ y su enfoque hacia el documental y la antropología; los de Montserrat Jurado Martín y Alberto Nieto Martín (2014) sobre la función de los festivales de cine como plataforma de los nuevos realizadores y el mapa de festivales de cine en España; el trabajo de Sedeños Valdellós (2013, p. 295) que destaca el valor de los festivales de cine para la globalización y transnacionalidad en el cine; y por supuesto las aportaciones de Rosana Vivar Navas (2016), entorno al aspecto lúdico de los festivales de cine, sus audiencias y su relación con los medios de comunicación.

En el ámbito concreto de la cinematografía, la labor del director de fotografía y el universo de posibilidades técnicoexpresivas que dispone para contar historias, queda expresado en Cortés-Selva (2018). No obstante, en el ámbito concreto de los festivales especializados en dirección de fotografía cinematográfica, destaca la reciente publicación focalizada en el análisis de las estrategias para la promoción de la fotografía cinematográfica, los estilos fotográficos y el desarrollo profesional del festival Camerimage (CORTÉS-SELVA; JURADO MARTÍN; OSTROVSKAYA, 2018).

Los motivos por lo que se decide organizar un festival de cine son diversos y de variada naturaleza, en consonancia con 
los intereses que pretenden cubrir sus organizadores, que cambian en función de si se trata de asociaciones culturales o entes más cercanos a la política o la empresa. La realidad es que en numerosas ocasiones $^{2}$ se establece una simbiosis entre ambos de cara a la celebración de un festival. Los entes culturales organizan el evento, mientras que los económicos y/o políticos aportan los fondos. Los patrocinadores pueden o no tener interés por ese fenómeno cultural, pero en cualquier caso se establece una relación subordinada y dependiente de los organizadores hacia los patrocinadores. Si la relación equilibra intereses, el evento progresa y se consolida, pero si no es el caso existen muchas posibilidades de que deje de celebrarse (JURADO MARTÍN, 2018, p. 144).

No están claros los motivos que llevan a la desaparición de un evento cultural y para cada caso habrá que observar su singularidad. Es un fenómeno más complejo que una actividad cultural aislada, pero su capacidad de acción no ha sido aprovechada por sus organizadores (GUILLOT; LLORENS, 2014, p. 8), exceptuando algunos que han logrado una firma dentro y fuera de España. En este grupo pueden nombrarse festivales como el de San Sebastián, Seminci de Valladolid, Sitges, Cinema Jove, entre otros. El número de festivales ha sido cambiante, desde el boom de los noventa, pasando por la crisis de la primera década de este siglo (JURADO MARTÍN; NIETO MARTÍN, 2014, p. 101) y,

2 Datos extraídos de Festimapp 2014: http://ivac.gva.es/foment/ festimapp/informe. Los festivales de cine de este estudio se caracterizan por estar promovidos por organizadores privados, en su gran mayoría organizados por una persona durante todo el año, aunque son más durante la celebración del evento, y financiados con fondos públicos, o mixtos entre públicos y privados. 
de nuevo, un tímido resurgir (JURADO MARTÍN, 2017, p. 252) Entonces, ¿qué es lo que determina que un certamen se consolide o que deje de celebrarse? Son muchas las posibilidades de estudio a partir de esta reflexión. Una vía es localizar la clave del éxito en un certamen con reconocida reputación y otra buscar los motivos que han contribuido a su desaparición.

Así es como este artículo toma como referencia el festival extinto Madridimagen. El certamen logró consolidarse como el gran festival de cine especializado en fotografía en el ámbito español. Entre 1996 y 2012 se celebraron un total de 17 ediciones en las que fueron premiados jóvenes autores que hoy en día gozan de gran reputación en su sector.

Este artículo pretende rescatar la historia del certamen a partir de los contenidos web que todavía pueden encontrarse diseminados, demostrar la importancia de su función en la consolidación de los profesionales en la dirección de fotografía, así como analizar los posibles motivos que determinaron su fin. La metodología será el estudio de caso, sobre el que se desarrollará el análisis documental a partir de la web y el análisis biográfico sobre los expertos premiados y su consolidación profesional hasta la actualidad.

\section{Marco de Los Festivales de Cine}

\section{Los festivales de cine: rasgos definitorios}

Existen investigaciones centradas en una o varias funciones o descripciones y respaldan así un certamen cinematográfico como un evento de gran envergadura (BONET, 2011, p. 2) que 
implica la exhibición audiovisual (CABEZÓN; GÓMEZ-URDÁ, 1999, p. 192), la celebración de un concurso y favorece el mercado al tratarse de encuentros profesionales (LINARES PALOMAR, 2009 , p. 175), así como el encuentro entre profesionales y noveles (ADELMAN, 2005, p. 187-189). Los festivales fomenta la distribución (MATAMOROS, 2009, p. 190), la promoción del audiovisual (MIÑARRO, 2013, p. 126), una programación no comercial (AAS, 1997, p. 55), apoyan el desarrollo artístico e implican el respaldo comunitario de las instituciones interesadas (INKEI, 2005, p. 7-9), lo que desemboca en ocasiones en cubrir la función de ser promotor turístico de una ciudad (ELIZAGARATE, 2008, p. 140); y finalmente, son un foco mediático para la promoción de las entidades que lo organizan (HARBORD, 2002).

El estudio de los festivales de cine se convierte en un ejercicio complejo por las variaciones en su número a lo largo del tiempo. El censo de festivales de cine del año 2000, los fijaba en más de 200 (JURADO MARTÍN, 2017, p. 245), y prudentemente la autora afirmaba que no reflejaba un panorama completo, ya que el proceso de inmersión en Internet y la posibilidad de contar con página web todavía no estaba tan extendido. En 2013 (JURADO MARTÍN; NIETO MARTÍN, 2014) el censo había disminuido considerablemente, aunque no supuso un estudio en relación a la situación económica de España, sí se planteaba la relación de ésta con el descenso. Ese mismo año, en el censo Festimapp (GUILLOT; LLORENS, 2014, p. 7) se estimaba el descenso -sólo en el ámbito de la comunidad autónoma valenciana- en un $25 \%$. Los autores insinúan que todavía podría haber sido más dramático si no hubiera sido por la tendencia a la especialización. "En la especialización 
parece estar la supervivencia y el éxito de los nuevos festivales" (JURADO MARTÍN, 2017, p. 252, 254), en un contexto caracterizado por "una crisis económica que causó estragos en la inversión en cultura a finales del siglo XX e inicios del XXI".

No obstante, han sido diversas las propuestas entorno a los elementos que se pueden fomentar de cara a la consolidación de un certamen: el fomento de presencia prolongada a lo largo del año, potenciar su valor de mercado, cofinanciar obras en curso, publicaciones especializadas, y cultivar la relación con la Administración Pública, "que aparecen a menudo a ojos de los festivales como meros cajeros automáticos proveedores de fondos (GUILLOT; LLORENS, 2014, p. 11-14). Esta última propuesta estrecharía lazos con las entidades y le otorgarían más consolidación. Se trata de un enfoque que otorgaría a los festivales de mayor protagonismo en el ámbito de la industria cultural y no como mera actividad cultural.

\section{Los festivales de cine: entre la industria cultural, la creación audiovisual y la especialización periodística}

El estudio de los festivales de cine se encuentra ubicado en dos marcos de referencia: desde el ámbito económico como fenómeno de las industrias culturales; desde el ámbito de la producción audiovisual, en el de la creación audiovisual; y en el ámbito del periodismo, en el de la especialización periodística.

En el primer caso, las industrias culturales, también denominadas economías creativas (ZALLO, 2011, p. 155) o su versión más moderna, industrias creativas (GARNHAM, 2011, p. 21), deben su origen a los estudios de Adorno y Horkheimer 
(1947/2005) y hacen referencia a la estandarización de las técnicas de distribución no implicando las de producción. La UNESCO (1982 apud TREMBLAY, 2011, p. 54) concreta que "las industrias culturales son los bienes y servicios culturales producidos, reproducidos, almacenados o difundidos según criterios industriales y comerciales”. Según Tremblay (2011, p. 57-58) su contenido abarca la producción y distribución de libros, diarios, discos, programas, películas, y emisiones de radio y televisión.

Son pocos los estudios que incluyen a los festivales de cine como eventos culturales, que al igual que los musicales, literarios o artísticos, deben contemplarse en este marco de referencia y en general la aplicación de medidas de política industrial al ámbito cultural es todavía bastante novedoso (ZALLO, 2011, p. 175). Al no generar realmente una industria desde el punto de vista económico, $\mathrm{y}$ quizás al no generar beneficios como pudieran hacerlo otros eventos en este sector, suelen acaparar menos atención para los investigadores y para los grupos con intereses empresariales o políticos.

En el segundo caso, desde el ámbito del periodismo, cubre intereses del ámbito de la difusión de una actividad cultural no necesariamente ligada al crecimiento económico, sino al crecimiento intelectual y su difusión. Los festivales de cine se estudian en la denominada especialización en periodismo cultural(RIVERA, 2003; RODRÍGUEZ PASTORIZA, 2006), y más específicamente en el periodismo cinematográfico (ESTEVE RAMÍREZ; FERNÁNDEZ DEL MORAL, 1999). Se trata de una especialización que hoy en día debe profundizar en los contenidos que difunde.

Según Esteve Ramírez y Fernández del Moral (1999, p. 131), el redactor especializado en el ámbito cultural ayuda a 
comprender una obra literaria, valora la calidad de una película, orienta sobre el valor de una exposición u obra pictórica, etc., pero generalmente cuando escribe o habla sobre los certámenes cinematográficos se centra en las actividades que se desarrollan en él, pero no analiza la función del evento. Es decir, promociona las producciones, actividades, personas, etc., que el certamen pone a su disposición, pero no al certamen en sí mismo, por lo que no otorga al espectador argumentos que le permitan tener un juicio crítico para valorar la actividad cultural que desarrolla.

En consecuencia, tampoco pone en valor sus posibilidades dentro de las industrias culturales, al no destacar su potencialidad a nivel económico como pudiera ocurrir en un fenómeno musical o literario. La ausencia de estos intereses extra culturales restan posibilidades de consolidación y crecimiento en el sector de la industria audiovisual y creativa de los festivales de cine, alejándolos de tener la misma consideración de un macro concierto musical alternativo o un concurso literario tipo Premio Planeta.

\section{Objetivos y Metodología}

Este estudio pretende rescatar y difundir la historia inédita del extinto certamen Madridimagen: el único festival especializado en dirección de fotografía en España. Esta circunstancia despierta el interés por conocer el motivo por el que dejó de celebrarse y si como festival cumplió con una función básica de estos, servir de promotor para los creadores que premiaron. El segundo objetivo, consiste en conocer los motivos que provocaron su extinción analizando los factores determinantes de su historia, buscando aquellos elementos 
que den pistas sobre su declive. El tercer objetivo tratará de conocer si cumplió como promotor de los autores premiados, a tal efecto se analizará la producción audiovisual de sus galardonados.

Para el primer y segundo objetivo, rescatar la historia inédita y dibujar los elementos que supusieron su final, se ha recurrido al método documental ciñéndose a la búsqueda en la web ya que en el contexto de los festivales de cine, si no está en Internet prácticamente se puede afirmar que no existe. Por otra parte, es evidente la intención de borrar las huellas del certamen, ya que el festival MiM Series, organizado por la misma asociación de Madridimagen, emplea el mismo dominio web y eliminaron todo rastro de ediciones anteriores. No obstante, se localizó un documento en formato pdf correspondiente a uno de los catálogos, copiando el mismo enlace y cambiando la fecha que figura en éste, pudieron rescatarse los catálogos de otras tres ediciones, pero este método no obtuvo resultados positivos en el resto. Igualmente, y rescatando las noticias difundidas en su día por los medios de comunicación, se pudo reunir el palmarés de 14 de sus 17 ediciones.

Para el tercero de los objetivos, conocer la producción audiovisual de los autores premiados $y$, en concreto, los galardonados en la dirección de fotografía en cine, se ha recurrido al análisis biográfico empleando como fuente la base de datos especializada en el sector, $\mathrm{Imdb}^{3}$. La muestra de estudio se ha centrado en los premiados en las categorías a la Mejor Fotografía de Largometraje y Mejor Fotografía de Cortometraje ${ }^{4}$, las dos categorías más

3 Web: http://www.imdb.com/

$4 \mathrm{El}$ certamen fue generoso en la variedad de sus categorías, llegando premiar hasta 19 diferentes. A lo largo de sus 17 ediciones, 
emblemáticas del certamen. En el primer grupo se ha analizado la biografía de 17 autores, y en el segundo, 13. La búsqueda en Imdb se ha centrado en los puestos profesionales ocupados por producto audiovisual independientemente de si se trata de programa para televisión, capítulo de serie, largometraje, cortometraje, etc., y figura con más de un crédito en el mismo producto. Por este motivo, los resultados de la producción son mayores en los autores que han producido series de televisión con cierta constancia. Por lo tanto, se trabaja con un análisis cuantitativo.

\section{El certamen Madridimagen: cuando la política pesa sobre la cultura}

Madridimagen conserva poco contenido en la web. A pesar de ser un certamen que nació a finales del siglo $\mathrm{XX}$, coincidiendo con el gran auge de los festivales de cine en España, y donde muchos ya aprovechaban las posibilidades de Internet para promocionarse, en la actualidad, no se han hallado prácticamente contenidos sobre los primeros años del certamen. El evento nace en 1996 y, 13 años después, en su presentación de 2008, sus organizadores aseguran de él que es un festival que apuesta por la calidad fotográfica y la innovación en las producciones, da un valor especial a los cortometrajes y reconoce el compromiso de sus espectadores. Destaca su vocación por el "arraigo profesional y de proyección social y cultural".

algunas desaparecieron y otras se crearon. Hubo una categoría a la Mejor Dirección de Fotografía para Cortometraje, pero no fue constante y su medición supone obtener resultados incompletos. Por otra parte, también premió la imagen fotográfica, pero en este ámbito fueron numerosas las subdivisiones premiadas. 
El catálogo de 2008 es el más antiguo que todavía puede encontrarse en la web junto con los de 2009, 2011 y 2012, las más significativas en lo que tiene que ver con el desenlace. Cuenta con una sección competitiva que proyecta en sus salas y también tiene secciones paralelas como el Golden Award, para rendir homenaje por su trayectoria a un reconocido profesional de la dirección de fotografía, ciclos de películas, mesa de redonda para el estudio en relación a la fotografía y seminarios de especialización. Todas estas actividades sirven para reconocer el valor añadido de un festival y no de un concurso o muestra de cine no competitiva.

Se aprecia también su preocupación por la formalidad en tanto que difunde el perfil y nombre de los componentes del jurado, siendo un total de $5 \mathrm{y}$ dos de ellos directores de fotografía. Todos tienen un completo perfil profesional en el sector y la única crítica es la ausencia de equilibrio en términos de igualdad que pueda enriquecer el enfoque del jurado. En competición hay tanto largometrajes como cortometrajes y diferentes categorías: largometraje internacional y ópera prima, ambos para el largometraje; y varios premios para el formato en cortometraje, donde compiten también las escuelas de cine.

En 2008, el festival estaba organizado por la Asociación Cultural Madridimagen. Figuraban como patrocinadores oficiales el Ministerio de Cultura ICAA, la Concejalía de Cultura y Deportes de la Comunidad de Madrid y el Área de las Artes del Ayuntamiento de Madrid. Entre sus más de 60 colaboradores el certamen destaca la labor del Círculo de Bellas Artes, Filmoteca Española y Sony España.

En esa edición estuvo coordinador por Sergio Prado y nueve personas más completaron el equipo, además de César Martínez como presidente de Madridimagen, Luis Villar como 
vicepresidente y Roberto Lázaro como secretario. En 2009 esta estructura se mantiene prácticamente igual, añadiendo a Tote Trenas en el equipo organizador en lugar de asesor.

En el catálogo de 2009 se aprecian que sus contenidos fueron los mismos, aunque sus organizadores critican la falta de apoyo de las instituciones en cuanto a los recursos que reciben y su motivación por seguir adelante a pesar de esta situación con "un espíritu combativo". En un contexto político en el que se negaba la crisis, ellos escriben que es evidente y que no quieren sumarse al sentimiento de tristeza:

No hemos podido resistirnos a la tentación de usar el viejo título del LP homónimo de Supertramp para la presentación de este año: Crisis ¿Qué crisis? Aunque el sector se encuentra en una clara situación de crisis, por mucho que algunos se empeñen en negarlo con cifras y estadísticas, (iqué pregunten a los profesionales y a las empresas y se les desmontarán los números!) en Madridimagen no hemos querido contagiarnos de tan pesimista situación. La producción ha disminuido y con ello la inversión en eventos y publicidad, por lo tanto, las iniciativas divulgativas como este festival, han visto disminuido sus recursos. Pero no nos vamos a lamentar, a mal tiempo buena cara.

En esta edición se suma un miembro más al jurado convirtiéndose en número par, todos ellos con perfil nacional y ninguna mujer entre sus nombres. Siguen manteniendo, a pesar de las dificultades económicas por las que afirman pasar, la celebración de mesas redondas y conferencias y sus ciclos no competitivos. Sin embargo, no figuran patrocinadores, sino un listado de anunciantes, donde el ICAA, la Comunidad de Madrid, y el Ayuntamiento de 
Madrid aparece como el resto de anunciantes.

En 2011 los patrocinadores oficiales son los mismos que los de 2008, cuatro entidades colaboradoras destacadas y otras 51 colaboradores entre empresas y personas físicas. El equipo organizativo se mantiene, el jurado está compuesto por cinco personas - una de ellas, la actriz Esperanza Pedreño- y dos son directores de fotografía.

El texto de presentación del catálogo está entre la prudencia hacia los patrocinadores y la necesidad de ser diplomáticos por su dependencia económica y ante un presupuesto ya de por sí bajo, tal y como se deduce por expresiones como la que sigue: "Madridimagen sobrevive año tras año a las turbulencias propias de nuestro tiempo, para desarrollar su tarea como difusor de propuestas cinematográficas de alto grado". Se diluye el "espíritu combativo". Sus organizadores aseguran que:

Elrespaldoyelrespeto delos distintospatrocinadores oficiales hacia el comité organizador ha sido una constante a lo largo de estos años. La independencia de Madridimagen no ha sido hasta ahora objeto de debate. Queremos manifestarles nuestro más sincero agradecimiento por su confianza.

El catálogo de 2012 cambia de diseño pero el certamen sigue manteniendo sus mismos contenidos. El Ministerio de Cultura, a través del ICAA, deja de figurar como patrocinador oficial y se mantienen la Comunidad de Madrid y el Ayuntamiento de Madrid. Se mantienen las mismas entidades colaboradoras, los miembros de su organización y el número de colaboradores, aunque sigue siendo numeroso, se reduce a 31 . 
Sus organizadores presentan como novedad la sección de Óperas Primas, ampliándose también a obras procedentes de Iberoamérica. El premio pasa a denominarse 'Dama', destacando así a su nuevo patrocinador oficial, que se suma a los existentes: Comunidad de Madrid y Ayuntamiento de Madrid. Repiten intenciones y prácticamente sus palabras en lo relativo a las turbulencias y tiempo de crisis y a su incansable entusiasmo por el proyecto que llevan adelante. Su jurado está compuesto por 5 integrantes, dos de ellos mujeres, una guionista y otra productora.

En 2013, en la que sería la decimoctava edición del festival, se inicia una nueva sección destinada a la ficción televisiva. Numerosos medios se hacen eco de la noticia en lo que se describe como una edad de oro de la televisión en España. El certamen sigue premiando escuelas, cortometrajes, óperas primas y la novedad: largometrajes auto producidos.

Solo Dama aparece como patrocinador oficial, mientras que la Comunidad de Madrid y el Ayuntamiento de Madrid aparecen como patrocinadores junto con la Fundación AISGE, que repite como entidad colaboradora junto con otras cuatro. El equipo organizador se mantiene, se amplía el comité asesor y sus colaboradores llegan a los cuarenta y tres. Además del jurado de cine se crea el jurado para la valoración de las series. Ambos se integran por cinco personas, expertos en su ámbito, y en cada uno, dos de sus integrantes son mujeres.

Sin embargo, esta edición marcó un antes y un después porque, si bien fue considerada en ese momento una más en la historia de Madridimagen como festival especializado en fotografía, lo cierto es que sólo dos años después, este carácter especializado en fotografía 
cinematográfica desapareció, dejando exclusivamente un festival especializado en series, conocido hoy en día como MIM Series, y que se describe a sí mismo como "un festival único en España".

Su equipo organizador está integrado en gran parte por el original de Madridimagen que buscó la supervivencia en la captación de nuevos ingresos y que por motivos no declarados borraron toda huella de Madridimagen, también un festival único en España en su sector. Si bien es cierto que las posibilidades de especialización en esta nueva vertiente son factibles, ya que en ese momento no había ningún otro evento especializado en series, lo cierto es que tampoco lo había en el de la fotografía cinematográfica, ni tampoco había festivales que premiaran tan ampliamente este sector profesional. Aunque no son muchos los años que quedan olvidados en algún lugar en la web, los analizados han sido suficientes para conocer el deterioro y el desenlace final de Madridimagen.

Durante sus últimos años se mantuvo el equipo organizador, así como el número de miembros del jurado, aumentado poco a poco la presencia de la mujer, lo que supone una adaptación a la realidad del sector. Mientras contaron con los patrocinadores oficiales, se mantuvieron en activo, pero la desaparición del ICAAy poco después del resto de las instituciones públicas fue determinante para que dejara de celebrarse. En su intento por sobrevivir abrieron nuevas vías como la participación Iberoamericana, y al año siguiente la de las series de televisión, que terminó por sepultar definitivamente al único festival de cine especializado en fotografía en España.

Por la implicación y perfil de los patrocinadores oficiales del certamen, se estima oportuno establecer el paralelismo entre el contexto político y económico de España. Madridimagen nace 
coincidiendo con la llegada del Partido Popular al gobierno; su celebración se mantiene durante el gobierno del PSOE de Rodríguez Zapatero, que las gana en 2004. En 2009, tras el punto álgido que supone la crisis en España, Madridimagen afirma resentirse por los recortes en su presupuesto. No obstante, a pesar de esta situación, no es hasta con la toma de posesión del Partido Popular bajo la presidencia de Mariano Rajoy, en 2012, cuando pierde los ingresos del ICAA, y un año más tarde, 2013, los otros dos patrocinadores oficiales, Comunidad de Madrid y Ayuntamiento de Madrid, pasan a ser patrocinadores y solo Dama aparece como oficial. Poco después, 2013 se convierte en el año de referencia de la primera edición de MiM y Madridimagen, como festival de fotografía, cae en el olvido digital.

Imagen 1 - Paralelismo entre el contexto político en España y los momentos clave de Madridimagen

\begin{tabular}{|c|c|c|c|c|c|c|}
\hline 1996 & 2004 & 2008 & 2009 & 2011 & 2012 & 2013 \\
\hline $\begin{array}{l}\text { PP gana las } \\
\text { elecciones }\end{array}$ & $\begin{array}{l}\text { PSOE } \\
\text { gana las } \\
\text { elecciones }\end{array}$ & $\begin{array}{l}\text { Inicio } \\
\text { crisis } \\
\text { España }\end{array}$ & & $\begin{array}{l}\text { PP gana las } \\
\text { elecciones }\end{array}$ & $\begin{array}{l}\text { PP toma } \\
\text { posesión }\end{array}$ & \\
\hline $\begin{array}{l}\text { Nace } \\
\text { Madridimagen }\end{array}$ & & & $\begin{array}{l}\text { Madridimagen } \\
\text { confirma } \\
\text { problemas de } \\
\text { presupuesto, } \\
\text { "espíritu } \\
\text { combativo" }\end{array}$ & $\begin{array}{l}\text { Prudencia en la } \\
\text { relación con los } \\
\text { patrocinadores }\end{array}$ & $\begin{array}{l}\text { Último año de } \\
\text { Madridimagen } \\
\text { Patrocinadores: } \\
\text { Cae el ICAA, } \\
\text { aparece DAMA }\end{array}$ & $\begin{array}{l}\text { Desaparece } \\
\text { antigüedad } \\
\text { Madridimagen y } \\
\text { nace la primera } \\
\text { edición del MiM }\end{array}$ \\
\hline
\end{tabular}

Fuente: Elaboración propia. 


\section{Madridimagen y la función de consolidación de profesionales}

La cultura ha sido un sector castigado tras la crisis económica en España. Sin entrar en este debate, sí se quiere plantear desde este artículo, el valor formativo en el ámbito profesional de los festivales de cine y en concreto del que nos ocupa, el certamen Madridimagen. Sus organizadores insisten en el riesgo que asumen sus creadores cada vez que invierten en un proyecto, y el ejercicio personal por la innovación y la superación, para estar a la altura de los profesionales de su sector, no sólo en España sino en el ámbito internacional. En definitiva, los festivales son plataformas de ensayo-error, espacios formativos para los futuros profesionales de la industria cinematográfica, los que mueven el motor de la cultura y siembran la semilla del futuro industrial de todo un país.

En este contexto se va a analizar si Madridimagen cumplía con su función de promotor de los autores premiados y, en concreto, en las dos categorías más emblemáticas durante todas que ediciones, Mejor Fotografia en Largometraje y Mejor Fotografía en Cortometraje. En este análisis se valorará si tras el paso por Madridimagen, el creador ha experimentado un crecimiento en su producción y si entre los puestos ocupados lo fue en el ámbito de la dirección de fotografía.

Así y todo, los directores que finalmente se analizaron son los que se concretan en la siguiente tabla, que supone un extracto del palmarés. 


\section{Imagen 2 - Palmarés Madridimagen en las categorías analizadas}

\begin{tabular}{|c|c|c|}
\hline & Mejor Fotografía Largometraje (*) & $\begin{array}{l}\text { Mejor Fotografía } \\
\text { de Cortometraje }\end{array}$ \\
\hline $1996\left(1^{\mathrm{a}}\right)$ & Juan Ruíz Anchía por LORCA & \\
\hline $1997\left(2^{a}\right)$ & $\begin{array}{l}\text { Rogier Stoffers por CHARACTER; } \\
\text { Martin Langer por } 14 \text { DAYS TO LIFE }\end{array}$ & $\begin{array}{l}\text { ESPOSADOS } \\
\text { de Juan Antonio } \\
\text { Castaño (Spain) }\end{array}$ \\
\hline $1998\left(3^{\mathrm{a}}\right)$ & $\begin{array}{l}\text { Theo Bierkens por WHEN THE LIGHT COMES; } \\
\text { Vittorio Storaro (AIC, ASC) por TANGO } \\
\text { Jens Fischer (FSF) por BENEATH THE SURFACE }\end{array}$ & $\begin{array}{l}\text { THE } \\
\text { ABANDONED } \\
\text { ROAD (Noruega) } \\
\text { de Morten } \\
\text { Skallerud }\end{array}$ \\
\hline $1999\left(4^{a}\right)$ & Julian Elizalde, por JAZZOO & $\begin{array}{l}\text { OBSESION, de } \\
\text { Carlos Esteban }\end{array}$ \\
\hline $2000\left(5^{\mathrm{a}}\right)$ & Gaizka Bourgeaud, por TORRE & $\begin{array}{l}\text { EN MALAS } \\
\text { COMPAÑIAS, de } \\
\text { Antonio Hens }\end{array}$ \\
\hline $2001\left(6^{a}\right)$ & Alfonso Parra por MUJERES EN UN TREN & $\begin{array}{l}\text { MUJERES EN UN } \\
\text { TREN de Jorge } \\
\text { Torregrosa }\end{array}$ \\
\hline $2002\left(7^{a}\right)$ & David Carretero por MALA ESPINA & $\begin{array}{l}\text { NADA QUE } \\
\text { PERDER, de Rafa } \\
\text { Russo }\end{array}$ \\
\hline $2003\left(8^{\mathrm{a}}\right)$ & Paco Belda por CICLO & $\begin{array}{l}\text { EN CAMAS } \\
\text { SEPARADAS, de } \\
\text { Javier Rebollo }\end{array}$ \\
\hline $2004\left(9^{a}\right)$ & Dario J. Ferrer por GRIS & $\begin{array}{l}\text { FISICA II de } \\
\text { Daniel Sánchez } \\
\text { Arévalo }\end{array}$ \\
\hline $2007\left(12^{a}\right)$ & David Tudela por JUEGO & $\begin{array}{l}\text { MENSAJES DE } \\
\text { VOZ de Fernando } \\
\text { Franco }\end{array}$ \\
\hline $2008\left(13^{a}\right)$ & Álvaro Gutiérrez por VIAJE A BANGKOK & $\begin{array}{l}\text { HETEROSEXUALES } \\
\text { Y CASADOS de } \\
\text { Vicente Villanueva }\end{array}$ \\
\hline $2010\left(15^{\mathrm{a}}\right)$ & Luis Bellido por EL IDIOMA IMPOSIBLE & $\begin{array}{l}\text { LA VENTAJA } \\
\text { DEL SICARIO } \\
\text { de Luis Moreno }\end{array}$ \\
\hline $2011\left(16^{a}\right)$ & Sergi Vilanova por LA CULPA & $\begin{array}{l}\text { EL SOMRIURE } \\
\text { AMAGAT de } \\
\text { Ventura Durall }\end{array}$ \\
\hline $2012\left(17^{\mathrm{a}}\right)$ & Gorka Gómez, por MONSTERS DO NOT EXIST & $\begin{array}{l}\text { DESAYUNO CON } \\
\text { DIADEMA de } \\
\text { Óscar Bernàcer }\end{array}$ \\
\hline
\end{tabular}

Fuente: Elaboración propia. (*) A partir de 1999 se denomina Mejor Director de Fotografía Novel; a partir 2012: Mejor Fotografía Novel 
Los resultados del estudio revelan que, tanto los premiados en la categoría de Mejor Fotografia Largometraje, como la de Mejor Fotografía Cortometraje experimentaron un incremento de su producción audiovisual después del reconocimiento recibido en Madridimagen, más evidente en el caso de los cortometrajes. Mientras que el primer grupo experimentó un crecimiento del $8 \%$, el segundo lo hizo en un $41,5 \%$.

Imagen 3 - Roles profesionales ejercidos por los autores, antes y después de ser premiados en Madridimagen

\section{Roles profesionales ejercidos por los directores antes y después de ser premiados en Madridimagen}

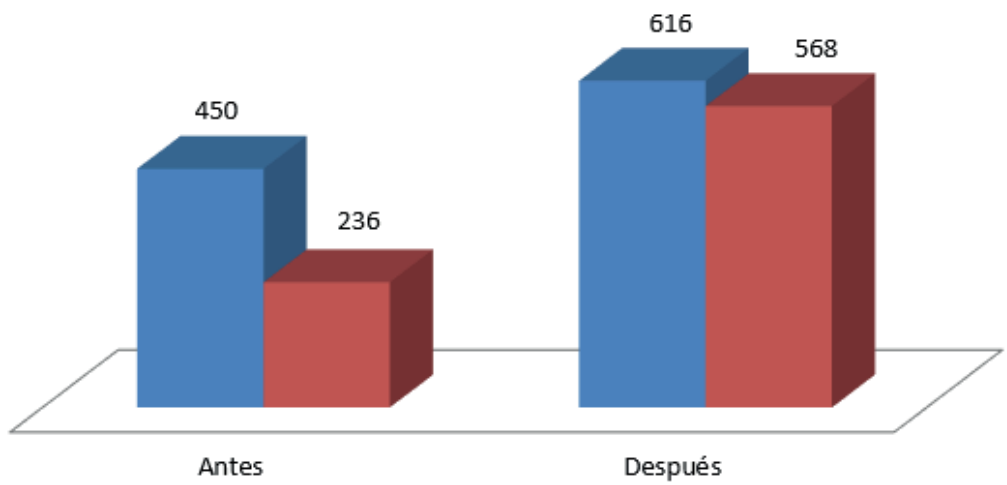

Fuente: Elaboración propia

Cabría pensar que la producción de los premiados en el ámbito del corto es mucho mayor que la de los premiados por sus largometrajes. Analizando en detalle, se observa una doble tendencia en el método de premiar del jurado de Madridimagen 
que desemboca en la posible creencia de que los directores de largometrajes tienden a tener menos éxito profesional que el segundo grupo.

En el caso de los premiados por la Mejor Fotografía en Largometraje, en las primeras ediciones existe una predisposición a premiar a reconocidas figuras de la dirección de fotografía con una extensa trayectoria, lo que implica mayor currículo previo al premio recibido en Madridimagen. Además, al tratarse de autores consolidados y, en algún caso, veteranos, su producción posterior se mantiene más o menos constante o inicia un descenso paulatino, como es el caso de Juan Ruiz Anchía o Vittorio Storaro. En otros casos se dispara exponencialmente, como es el caso de Paco Belda, Gaizka Bourgeaud o David Tudela; y destacar el caso de Julián Elizalde, que de no contar con ninguna producción en la que figurara como director de Fotografía anterior a Madridimagen, a partir de ese momento se dispara hasta superar las 70 películas con este perfil. Otra característica de los premiados en esta categoría fue la especialización en el desempeño de los roles en dirección de fotografía o cámara, antes y después de su paso por el certamen. 
Imagen 4 - Puestos profesionales de los directores antes y después del reconocimiento en Madridimagen. Madridimagen.

\begin{tabular}{|c|c|c|c|c|c|c|c|}
\hline & & \multicolumn{3}{|c|}{ ANTERIOR } & \multicolumn{3}{|c|}{ POSTERIOR } \\
\hline $\begin{array}{l}\text { Mejor Fotografia } \\
\text { en Largometraje }\end{array}$ & \begin{tabular}{|l|} 
Año en \\
Madridim \\
agen \\
\end{tabular} & $\begin{array}{l}\text { Producción } \\
\text { anterior }\end{array}$ & \begin{tabular}{|l|}
$\begin{array}{l}\text { Otros } \\
\text { créditos }\end{array}$ \\
\end{tabular} & TOTAL & $\begin{array}{l}\text { Producción } \\
\text { posterior }\end{array}$ & \begin{tabular}{|l|}
$\begin{array}{l}\text { Otros } \\
\text { créditos }\end{array}$ \\
\end{tabular} & TOTAL \\
\hline Juan Ruiz Anchía & \begin{tabular}{|r|}
1996 \\
\end{tabular} & $\begin{array}{r}50 \\
\end{array}$ & \begin{tabular}{|r|}
4 \\
\end{tabular} & 54 & 26 & \begin{tabular}{|r|}
4 \\
\end{tabular} & 30 \\
\hline Rogier Stoffers & 1997 & 31 & 4 & 35 & 36 & 6 & 42 \\
\hline Martin Langer & 1997 & 4 & 1 & 5 & 43 & 2 & 45 \\
\hline Theo Bierkens & 1998 & 41 & 19 & 60 & 53 & 5 & 58 \\
\hline Vittorio Storaro & 1998 & 73 & 36 & 109 & 34 & 26 & 60 \\
\hline Jens Fischer & 1998 & 29 & 2 & 31 & 21 & 9 & 30 \\
\hline Julián Elizalde & 1999 & 0 & 2 & 2 & 77 & 6 & 83 \\
\hline Gaizka Bourgeaud & 2000 & 3 & 3 & 6 & 42 & 19 & 61 \\
\hline Alfonso Parra & 2001 & 10 & 4 & 14 & 20 & 2 & 22 \\
\hline David Carretero & 2002 & 7 & 6 & 13 & 14 & 2 & 16 \\
\hline Paco Belda & 2003 & 5 & 5 & 10 & 24 & 6 & 30 \\
\hline Darío J. Ferrer & 2004 & 1 & 0 & 1 & 4 & 8 & 12 \\
\hline David Tudela & 2007 & 9 & 6 & 15 & 19 & 1 & 20 \\
\hline Álvaro Gutiérrez & 2008 & 14 & 14 & 28 & 31 & 14 & 45 \\
\hline Luis Bellido & 2010 & 12 & 5 & 17 & 6 & 2 & 8 \\
\hline Sergi Vilanova & 2011 & 7 & 7 & 14 & 5 & 2 & 7 \\
\hline Gorka Gómez & 2012 & 31 & 5 & 36 & 29 & 18 & 47 \\
\hline $\begin{array}{l}\text { Mejor Fotografia } \\
\text { en Cortometraje }\end{array}$ & & 327 & 123 & 450 & 484 & 132 & 616 \\
\hline $\begin{array}{l}\text { Juan Antonio } \\
\text { Castaño }\end{array}$ & 1997 & 3 & 1 & 4 & 19 & 78 & 97 \\
\hline Morten Skallerud & 1998 & 4 & 15 & 19 & 11 & 27 & 38 \\
\hline Carlos Esteban & 1999 & 0 & 12 & 12 & 0 & 2 & 2 \\
\hline Antonio Hens & 2000 & 0 & 10 & 10 & 0 & 45 & 45 \\
\hline Jorge Torregrosa & 2001 & 0 & 11 & 11 & 0 & 97 & 97 \\
\hline Rafa Russo & 2002 & 0 & 10 & 10 & 0 & 18 & 18 \\
\hline Javier Rebollo & 2003 & 0 & 10 & 10 & 0 & 31 & 31 \\
\hline $\begin{array}{l}\text { Daniel Sánchez- } \\
\text { Arévalo }\end{array}$ & 2004 & 0 & 56 & 56 & 0 & 93 & 93 \\
\hline Femando Franco & 2007 & 0 & 27 & 27 & 0 & 67 & 67 \\
\hline Vicente Villanueva & 2008 & 0 & 15 & 15 & 0 & 21 & 21 \\
\hline $\begin{array}{l}\text { Luis Moreno } \\
\text { Bernardo }\end{array}$ & 2010 & 0 & 33 & 33 & 0 & 14 & 14 \\
\hline Ventura Drrall & 2011 & 0 & 20 & 20 & 0 & 28 & 28 \\
\hline Óscar Bemàcer & 2012 & 0 & 9 & 9 & 0 & 17 & 17 \\
\hline & & 7 & 229 & 236 & 30 & 538 & 568 \\
\hline & & & & 686 & & & 1.184 \\
\hline
\end{tabular}

Fuente: Elaboración propia. 
En el caso de los premiados por la Mejor Fotografía en Cortometraje, el jurado otorga el premio a profesionales que ya destacaban por su producción en la dirección de fotografía. Es el caso de Juan Antonio Castaño y Morten Skallerud. A partir de la tercera edición, y aunque el premio sea para la dirección de fotografía, lo cierto es que ninguno de los premiados figura en los créditos como director de fotografía: todos son los directores de sus cortos.

Esa segunda tendencia a la que se hacía referencia en la forma de premiar del jurado, denota en este punto que se trata de profesionales que todavía están probando diferentes roles dentro de la producción, lo que aumenta el número de repeticiones en los créditos en una misma película y, en consecuencia, en la valoración que se le ha dado en este análisis, ya que si bien en el ámbito del largometraje los perfiles están más definidos, y hasta se deduce que pronto dejaron atrás la práctica del cortometraje, en el caso de los premiados por su cortometraje, se observa además que ocuparon gran variedad de puestos, como directores, ayudantes de dirección, productores, guionistas, editores, etc. Esta circunstancia incrementa el número de veces que son valorados por su producción.

Destacar el caso de Carlos Esteban, que después del reconocimiento, no vuelve a figurar en IMDB con ningún otro trabajo y el vertiginoso crecimiento productivo de Juan Antonio Castaño, Antonio Hens, Jorge Torregrosa, Javier Rebollo o Daniel Sánchez-Arévalo, en muchos casos por sus trabajos en producciones para televisión.

La consideración final de esta parte del estudio revela que la tendencia a premiar en la categoría de mejor largometraje 
se decantaba por perfiles que ya contaban con cierto bagaje en la dirección de fotografía o cámara, y por lo tanto, denota poco arriesgo o más bien la intención de un reconocimiento por la trayectoria. Por el contrario, no ocurría lo mismo en la categoría de los cortometrajes, donde se valoraba la excelencia fotográfica global de la película, sus directores no necesariamente tenían interés específico por el aspecto de la imagen. Madridimagen conoce esta situación ya que también contaba con una categoría denominada Premio Especial del Jurado a la Mejor Fotografía de Cortometraje, que otorgó en solo 7 de sus 17 ediciones, es decir, esta distinción a la mejor fotografía en cortometraje, no fue digna de reconocerse siempre.

\section{Conclusión}

Madridimagen fue el festival de referencia en España para el reconocimiento de la imagen, la fotografía y la dirección de fotografía. Llegó a premiar hasta 19 categorías diferentes durante sus 17 años de existencia, entre 1996 y 2012.

A pesar de su especialización y prestigio en el sector, las decisiones políticas sobre las que pendía su dependencia económica, determinaron su final. No lo fue tanto la crisis económica en España, ya que en el momento álgido de esta, a pesar de los recortes, siguió celebrándose; mientras que en las mismas circunstancias otros certámenes en la misma situación lograron su supervivencia en la especialización y las nuevas formas de participación en las plataformas de inscripción de trabajos que abarató costes.

Dado que sus premios no se quedaban desiertos por falta de participación de los autores, no queda justificado el viraje 
a una nueva categoría sobre series que eclipsara por completo a Madridimagen hasta hacerlo desaparecer prácticamente por completo en la web.

Este artículo rescata la historia de Madridimagen y demuestra que cumplía con su función de promotor de los autores, que aumentaron su producción y presencia tras el reconocimiento recibido en el certamen. Madridimagen, como industria cultural, fue un agente activo en la progresión y consolidación de sus trayectorias profesionales, lo que en definitiva fue una apuesta por la cultura cinematográfica de un país. Su desaparición no queda justificada desde el ámbito cultural ya que cumplía con sus objetivos, por lo que se puede concluir que su final estuvo relacionado con cuestiones extra culturales que tenían que ver con la relación con sus patrocinadores.

Las posibilidades de los festivales de cine como industria cultural están, todavía, por explotar hasta llegar a convertirse en referentes en su sector como ya lo hacen otras artes, como la música o la literatura. En este punto los certámenes que realmente respondan a las exigencias del mercado asegurarán su permanencia con más firmeza.

\section{Referencias}

AAS, Nils Klevjer. Flickering shadow: quantifying the european film festival phenomenon. 1997. Disponible en: http://archive. is/20120604090843/www.obs.coe.int/online_publication/ expert/00001262.html. Aceso en: 10 abr. 2018.

ADELMAN, Kim. Cómo se hace un cortometraje. Barcelona: Robinbook, 2005. 
ADORNO, Theodor W; HORKHEIMER, Max. La raison dialectique. París: Gallimard, 2005. (Primera publicación de 1947).

BONET, Lluis. Tipologías y modelos de gestión de festivales. En: BONET, Lluis.; SCHARGORODSKY, Héctor (ed.). La gestión de festivales escénicos: conceptos, miradas, debates. Barcelona: Gescènic, 2011. p. 41-87.

CABEZÓN, Ángel; GÓMEZ-URDÁ, Félix. La producción cinematográfica. Madrid: Cátedra, 1999. (Colección Signo e Imagen).

CORTÉS-SELVA, Laura. Comunicación visual: fotografía cinematográfica avanzada. Barcelona: UOC, 2018.

CORTÉS-SELVA, Laura.; JURADO MARTÍN, Montserrat; OSTROVSKAYA, Liudmila. Camerimage, un festival único dedicado al arte de la fotografía cinematográfica. Revista Latina de Comunicación Social, México, v. 73, p. 614-632, 2018. Doi: 10.4185/RLCS-2018-1272.

DE VALCK, Marijke. Film festivals: from European geopolitics to global cinephilia. Amsterdam: Amsterdam University Press, 2007.

DE VALCK, Marijke; KRENDELL, Brendan; LOIST, Skadi. Film festivals: history, theory, method, practice. New York: Routledge, 2016.

ELIZAGARATE, Victoria. Marketing de ciudades: estrategias para el desarrollo de ciudades atractivas y competitivas en un mundo global. Madrid: Pirámides, 2008.

ESTEVE RAMÍREZ, Francisco; FERNÁNDEZ DEL MORAL, Javier. Áreas de especialización periodística. Madrid: Fragua, 1999.

GARNHAM, Nicholas. De las industrias culturales a las creativas. 
En: BUSTAMANTE RAMÍREZ, Enrique (Coord.). Industrias creativas: amenazas sobre la cultural digital. Barcelona: Gedisa, 2011. p. 21-48.

GUILLOT, Eduard; LLORENS, Pablo. Festimapp. Valencia:

Culturart, 2014. Disponible en: http://ivac.gva.es/fomento/ festimapp/informe. Aceso en: 10 abr. 2018.

HARBORD, Janet. Film festivals: media events and the spaces of flow. En: HARBORD, Janet. Film cultures. London: Sage, 2002. p. 5975.

INKEI, Peter. Apoyo festivales artísticos y culturales. Budapest: Budapest Observatory, 2005.

IORDANOVA, Dina.; PEER, Stephanie Van de (ed.) Film Festival Yearbook 6: film festivals and the middle east. St. Andrews: St. Andrews Film Studies, 2014.

IORDANOVA, Dina; CHEUNG, Ruby (ed.). Film festival yearbook 2: film festivals and imagined communities. St. Andrews: St. Andrews Film Studies, 2010.

IORDANOVA, Dina; CHEUNG, Ruby (ed.). Film festival yearbook 3: film festivals and East Asia. St. Andrews: St. Andrews Film Studies, 2011.

IORDANOVA, Dina; LESHU, Torchin (ed.). Film festival yearbook 4: film festivals and activism. St. Andrews: St. Andrews Film Studies, 2012.

IORDANOVA, Dina; RHYNE, Ragan (ed.). Film festival yearbook 1: the festival Circuit. UK: St. Andrews Film Studies, 2009.

JURADO MARTÍN, Montserrat. El germen del cine más amateur. En: LAZO, C. M. (coord.). Nuevas realidades en la comunicación 
audiovisual. Madrid: Tecnos, 2017. p. 245-260. (Colección: Ediciones Universitarias).

JURADO MARTÍN, Montserrat. Propuesta de categorización de festivales de cine. Estudio de caso en España. Miguel Hernández Communication Journal, Elche-Alicante, v. 9, n. 1, p. 131-160, 2018. Doi: http://dx.doi.org/10.21134/mhcj.v0i9.238

JURADO MARTÍN, Montserrat; NIETO MARTÍN, Alberto. Nuevas propuestas, viejos circuitos. El papel de los festivales de cine españoles en la consolidación de los nuevos realizadores. Secuencias. Revista de Historia del Cine, Madrid, n. 39, p. 100-122, 2014.

LINARES PALOMAR, R. La promoción cinematográfica. Estrategias de comunicación y distribución de películas. Madrid: Editorial Fragua, 2009.

MATAMOROS, Davd. Distribución y marketing cinematográfico. Barcelona: Universidad de Barcelona, 2009.

MIÑARRO, Laura. Cómo vender una obra audiovisual. Una aproximación a la distribución de los contenidos audiovisuales. Barcelona: Universitat Oberta de Cataluña, 2013.

NAVAS, Rosana Vivar. Los festivales de cine en la era de los new media: una perspectiva lúdica sobre la fiesta del cine y sus públicos. 2016. Tesis (Doctoral) - Universidad de Granada, Granada, España, 2016.

RIVERA, Jorge. El periodismo cultural. Buenos Aires, Argentina: Paidós, 2003.

RODRÍGUEZ PASTORIZA, Francisco. Periodismo cultural. Madrid: Síntesis, 2006. 
SEDEÑO VALDELLÓS, Ana María. Globalización y transnacionalidad en el cine: coproducciones internacionales y festivales para un cine de arte global emergente. Fonseca, Journal of Communication, Salamanca, n. 6, p. 296-315, 2013. Disponible en: http://revistas.usal.es/index.php/2172-9077/article/view/12129. Aceso en: 10 abr. 2018.

STRINGER, Julian. Regarding Film Festivals: Introduction. En: IORDANOVA, Dina. (ed.). The film festival reader. St. Andrews: St. Andrews Film Studies, 2013. p. 59-68.

TREMBLAY, Ginette. Desde la teoría de las industrias culturales. Evaluación crítica de la economía creativa. En: BUSTAMANTE, Enrique. (Coord.). Industrias creativas: amenazas sobre la cultural digital. Barcelona: Gedisa, 2011. p. 49-76.

TURAN, Kenneth. Sundance to Sarajevo: Film Festivals and the World They Made. Berkeley: University of California Press, 2002.

VALLEJO, Aida Vallejo. Festivales cinematográficos. En el punto de mira de la historiografía fílmica. Secuencias. Revista de Historia del cine, Madrid, n. 39, p. 13-42, 2014.

VALLEJO, Aida Vallejo. Festivales de cine documental: redes de circulación cultura en el este del continente europeo. 2012. Tesis (Doctoral) - Facultad de Filosofía y letras. Universidad Autónoma de Madrid, 2012. Disponible en: http://dialnet.unirioja.es/servlet/ tesis? codigo=35752. Aceso en: 10 abr. 2018.

ZALLO, Ramón. Estructura de la comunicación y de la cultura. Políticas para la era digital. Barcelona: Gedisa, 2011. 